\title{
Allergy to laboratory animals: a follow up study of its incidence and of the influence of atopy and pre-existing sensitisation on its development
}

\author{
P A Botham, C T Lamb, E L Teasdale, S M Bonner, J A Tomenson
}

\begin{abstract}
Objectives-To investigate the incidence of allergy to laboratory animals (ALA) during the first two years of employment, and to study the effect on ALA of atopy and sensitisation.

Methods-A follow up prospective study of ALA at the Zeneca (formerly ICI) Research Laboratories.

Results-The incidence of the disease during the first year of employment has remained at about $10 \%$ since the mid1980s. This compares with an incidence of $37 \%$ in the early 1980 s. The reduction in incidence and its maintenance at $a$ lower level is thought to be due to the introduction and management of improved engineering controls, working practices, and educational programmes designed to reduce exposure to allergens from laboratory animals. The underlying incidence of immunological sensitisation to animals (the presence of immunoglobulin E (IgE) antibodies to animal allergens) is much higher ( $40 \%$ after one and $53 \%$ after two years of exposure). Both atopic diathesis and presensitisation to laboratory animals increased the likelihood that a person would develop ALA. Conclusion-Neither factor predicted the disease accurately so their use should be restricted to the identification of people who may be more susceptible to the development of ALA (and thus who may need to pay particular attention to the use of personal protective equipment) rather than to their exclusion.
\end{abstract}

(Occup Environ Med 1995;52:129-133)

Zeneca Central

Toxicology

Laboratory, Alderley

Park, Macclesfield,

Cheshire

P A Botham

Zeneca

Pharmaceuticals, Alderley Park,

Macclesfield, Cheshire

C T Lamb

E L Teasdale

ICI Epidemiology

Unit, Brunner House,

Winnington,

Northwich, Cheshire

$S$ M Bonner

J A Tomenson

Correspondence to:

Dr P A Botham, Zenec

Dr P A Botham, Zen

Central Toxicology

Laboratory, Alderley Park,

Accepted 29 September 1994

Keywords: allergy to laboratory animals; atopy; sensitisation

The prevalence of allergy to laboratory animals (ALA) has been estimated with a variety of epidemiological techniques in studies over the past 20 years. A recent review of these studies found that the prevalence of ALA was in some cases as low as $11 \%-15 \%$, but in others it was $>30 \% .^{1}$ Subsequently, several other studies have confirmed this picture ${ }^{23}$ the most notable being a study of 5641 workers in Japanese animal laboratories where the prevalence was $23 \%{ }^{4}$

There have been far fewer studies of the incidence of ALA. At our own laboratories we have conducted two major prospective studies of ALA. In the first, $15 \%$ of 148 people developed ALA during their first year of employment. ${ }^{5}$ In the second, the "first year incidence" of ALA fell from $37 \%$ in people who started work with animals in 1980 or 1981 to $12 \%$ in those who started in $1984 .^{6}$ This reduction coincided with the introduction of new working practices and engineering controls designed to reduce potential exposure to allergens. A prospective study of 69 workers exposed to animals in a United States government research laboratory found a similar incidence $(13 \%){ }^{2}$

Two of these prospective studies also investigated the influence of one or more atopic indicators on the development of ALA. ${ }^{26}$ In both of these studies, it was concluded that the exclusion of people with atopy from working with animals could not be justified, as for every case of ALA that would theoretically be prevented, at least three people who would never develop the problem would also be denied employment.

At ICI (now Zeneca), since the mid-1980s there has been a coordinated process to try to maintain or reduce further the incidence of ALA by means of improved engineering controls and the use of personal protective equipment as well as by health surveillance and educational programmes. We report one outcome of this, namely the incidence of ALA during the first two years of employment in a group of 200 scientists and technicians recruited by ICI since the last study (from 1987-1990). The paper also considers the influence of atopy and of pre-existing sensitisation to laboratory animals on the development of ALA.

\section{Materials and methods} IMMUNOLOGICAL TESTS
Skin prick tests were conducted with eight animal allergens (guinea pig, mouse, rabbit, and rat urinary and dander extracts, prepared as described previously ${ }^{6}$ ).

Radioallergosorbent tests (RASTs) were conducted with guinea pig, mouse, and rat urine and rabbit dander. Skin tests were also conducted with group B2 (pollens), grasses $(2.5 \%)$, and Dermatophagoides pteronyssinus $(1 \cdot 2 \%)$ to assess atopy. Both methods were conducted as before ${ }^{6}$; a positive RAST test was one in which there was $\geqslant 4 \%$ binding of ${ }^{125}$ I-antihuman IgE; a positive skin test was one in which a wheal of $\geqslant 4 \mathrm{~mm}$ was recorded. A person was defined as atopic when they had a positive skin test to either 
Figure 1 Study design and subjects followed up during different stages of the study.

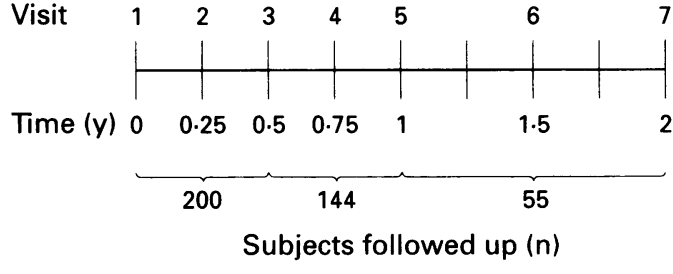

grass or house dust mite at the start of employment.

\section{SUBJECTS AND PROCEDURE}

The protocol for this study was approved by the ICI ethics committee. All people who were recruited to work with laboratory animals at our laboratories were asked to visit the medical centre as soon as possible after recruitment. Permanent and temporary staff were included. At this time (visit 1), each person was given a questionnaire that was completed with a member of the nursing staff. It asked if they had ever, as well as specifically during the past 12 months, had any allergic symptoms (see below) that may have been associated with exposure to animals, either in previous employment or from a household pet. Skin tests and RASTs were also conducted.

At three monthly intervals for the next year (visits 2-5) and then six monthly for a further year (visits 6-7), each volunteer was asked to return to the medical centre (fig 1 ). On each occasion a follow up questionnaire was completed. The information collected now included information on frequency of contact with animals, respiratory protection used (disposable mask or air fed helmet or hood) as well as the development or occurrence of allergic symptoms associated with exposure to animals at work. Skin tests and RASTs were repeated. Only those people who completed a minimum of six months of work with laboratory animals were included in the analyses. Of the 218 people whose data thus qualified for analysis, 200 were followed up during the first six months of the study, 144 were followed up from six months to one year and 55 were followed up during the second year (some people did not attend some of the early visits, but were followed up during the later part of the study).

Figure 2 Total number of cases of $A L A$ in study population and cumulative incidence of new cases of $A L A$ after different periods of follow up. The two cases of $A L A$ at the start of the study had sensitisation and symptoms at the start of the study that were later confirmed as $A L A$.

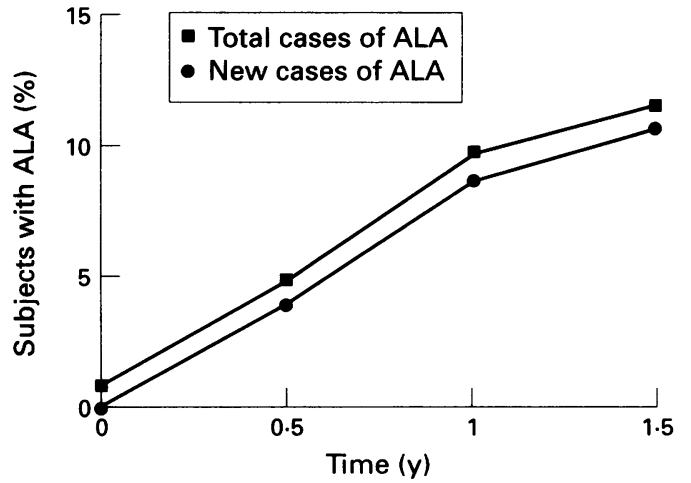

EVALUATION AND DIAGNOSIS OF SYMPTOMS Both the first and follow up questionnaires asked about any history of allergic symptoms of four categories (associated with exposure to animals); tightness of chest, or wheezing, or difficulty in breathing; eczema or allergic skin rashes; repeated attacks of sneezing, running, or blocked nose; and watery or itchy eyes. No attempt was made to rank the symptoms in terms of their severity.

A diagnosis of allergic to animals was made by the site Medical Officer (C L) after examination of the completed questionnaire and the results of the immunological tests. A person could be classified as allergic to animals either on the basis of a positive history and one or more positive tests (skin test or RAST) or positive history only. In reality, all people with a positive history also had a positive test.

\section{STATISTICAL ANALYSES}

Life table methods were used to calculate cumulative incidence at one year and two years. The cumulative incidence at one year was obtained as follows: cumulative incidence at one year $=$ cumulative incidence at six months $+((100-$ cumulative incidence at six months) $\times$ incidence at six months to incidence at one year). The cumulative incidence at two years was obtained in a similar manner. Incidences over the three periods, zero to six months, six months to one year, and one year to two years were derived from the subgroups of the study population that were followed up over those periods.

The accuracy of a predisposing factor was defined as follows: accuracy $=$ prevalence of factor $\times$ positive predictive value $+((100-$ prevalence of factor) $\times$ negative predictive value)

\section{Results}

INCIDENCE OF ALA

Figure 2 shows the results of the study. Of the 218 people who completed a minimum of six months work with animals, nine reported at the beginning of their employment that they had allergic symptoms associated with exposure to animals. These people were confirmed as sensitised to one or more laboratory species (guinea pigs, mice, rabbits, or rats), by skin prick tests or RASTs, or both. At the next two assessments, three months and six months after the start of employment, none of these nine could be confirmed as allergic to laboratory animals, as either their symptoms or their immunological status or both were not retained, but two were confirmed as having ALA at one year. None of the nine was followed up beyond a year and three were not followed up beyond six months.

At six months, a further eight people were confirmed as having developed ALA, giving an incidence of $4.0 \%$. Between six months and one year a further seven out of 137 people developed allergy (giving a cumulative incidence of $8.9 \%$ ). During the second year of the study, two out of 51 people developed ALA giving a cumulative incidence of $12 \cdot 4 \%$ at two 
Figure 3 Total number of sensitised people in the study population and cumulative incidence of new cases of sensitisation after different periods of follow up.

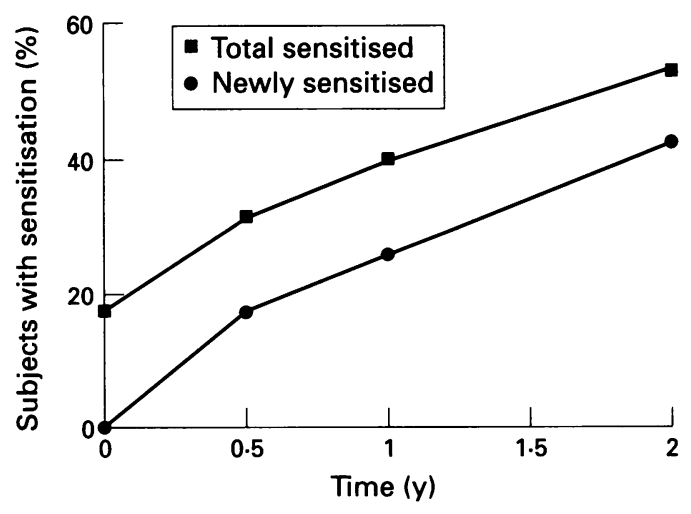

years and a total incidence of $13 \cdot 3 \%$.

Of equal interest was the finding (fig 3 ) of a high incidence of immunological sensitisation to laboratory animals. At the start of the study, $17 \cdot 4 \%$ of people were sensitised to one or more laboratory animals. At two years, a further $42 \cdot 1 \%$ had become sensitised giving a cumulative prevalence of $52 \cdot 9 \%$.

\section{PRE-EXISTING ATOPY AND SENSITISATION TO LABORATORY ANIMALS AS PREDISPOSING FACTORS IN ALA}

Figure 4 shows the relation between the development of ALA and the presence or absence of a positive skin test response to grass pollens or house dust mite extract at the start of employment (atopy) or the presence of immunoglobulin $\mathrm{E}$ (IgE) antibodies to one or more laboratory animal allergens at the start of employment. The nine people who were sensitised and symptomatic at the first visit were excluded from these analyses as there was strong evidence that these people were already allergic to animals (although only two were later confirmed to have ALA). Atopic people were significantly more likely to develop ALA or sensitisation to animals than non-atopic people, with increased relative risks of $7 \cdot 5$ at two years. Sensitised people were also much more likely to develop ALA, with an increased relative risk of 7.6 at two years. Combining atopy and pre-existing sensitisation did not increase the relative risks any further as only three people who were sensitised at the first visit were not atopic.

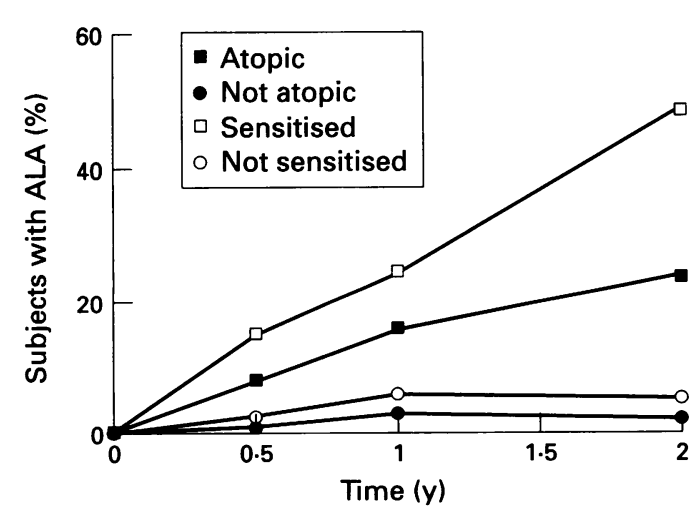

Figure 4 Relation between atopy (positive skin test to grass pollens or house dust mite) or sensitisation (presence of IgE antibodies) at visit 1 , and the development of $A L A$. Nine subjects who were sensitised and symptomatic at visit 1 are excluded.
Predictive value of predisposing factors in $A L A$

\begin{tabular}{lll}
\hline & \multicolumn{2}{l}{ Predictive value (\%) } \\
\cline { 2 - 3 } Predisposing factor & 1 Year & 2 Years \\
\hline Atopy & $62 \cdot 7$ & $66 \cdot 4$ \\
Sensitisation & 84.0 & $87 \cdot 4$ \\
\hline
\end{tabular}

If atopy or pre-existing sensitisation had been used as exclusion criteria it is possible to estimate the predictive value, that is, the number of atopic or previously sensitised people who developed ALA, and so would have been excluded from working with animals, and the number of non-atopic, non-sensitised people who did not develop the disease (table). These show that the exclusion of atopic people would have resulted in incorrect decisions for about one third of the population. The predictive value was increased to $87 \%$ if previously sensitised people were excluded. A combination of atopy and presensitisation did not increase the predictability of these factors for the development of ALA.

Figure 5 shows the relation between atopy at the start of the study and the development of sensitisation during the course of the study. Atopic people were much more likely to develop sensitisation (74.9\% at two years) than those who were not atopic (23.7\%).

\section{Discussion}

In a previous prospective study of ALA at ICI, the incidence of the disease during the first year of employment with laboratory animals was found to have fallen from $37 \%$ to $12 \%$ over a five year period. ${ }^{6}$ The only major change that had occurred during this time was the introduction of a code of practice for working with animals, which was designed to reduce potential exposure to allergens (by mandatory use of personal protective equipment and by extensive use of educational programmes). Some people were also monitored for a further two years but, although additional subjects developed ALA during this time, there was no evidence of an increase in the intensity of the symptoms and the overall incidence of asthma was only $2 \%$. Two thirds

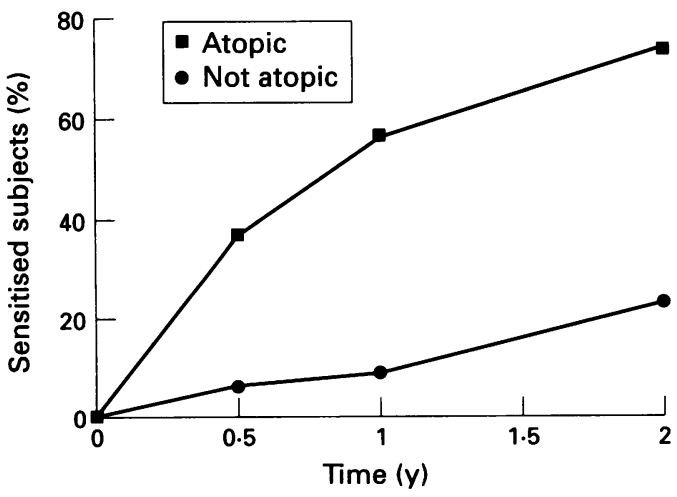

Figure 5 Relation between atopy and the development of sensitisation to laboratory animals. Thirty eight subjects who were sensitised at visit 1 are excluded. 
of those people who developed ALA during their first three years of employment did so during the first year. Our study has confirmed that the incidence of ALA at ICI has remained at about the same level and that most people seem to develop ALA during their first year of employment.

A weakness of our study was the decline in size of the study population, particularly after one year. The reduction in numbers of subjects followed up between six months and one year was due to the departure of employees on temporary contracts. The decline in study population between one year and two years was due to a number of factors, including lack of compliance with the study protocol, staff moving to positions that did not involve exposure to animals, and staff leaving employment. Only one person is known to have left the survey because of the development of ALA.

Nevertheless, taken together these studies suggest that ALA tends to develop in a mild form during the first few years of occupational exposure to animals, and that its incidence (and perhaps also its severity) can be controlled by the introduction of appropriate management controls. Neither study considered the effect of previous exposure to animals and there was no attempt made to measure actual or potential exposure to allergens. Although several groups have attempted to estimate exposure to animals by measuring concentrations of airborne allergens (recently reviewed by Gordon $e t a l$ ), interpretation and comparison of the data are very difficult. The techniques used to capture and measure the allergens have varied sufficiently to give rise to large (and probably, therefore, artefactual) variations in measured allergen concentrations. Also, there are many different allergens derived from animals. Thus, for example, in rat urine there are two main allergens; Rat $n$ IB (also known as $\alpha$ - $2 \mu$-globin) and Rat a IA (prealbumin).${ }^{8}$ In mouse urine, the main allergen is Agl (also called prealbumin) but another important allergen $\mathrm{Ag} 3$ is found in mouse dander. ${ }^{9}$ Allergen load in the atmosphere is also highly dependent on stock density, air circulation, and humidity and measurements of room concentrations of allergens can significantly underestimate the levels generated locally-for example, during the handling of an animal, and hence underestimate potential exposure. Therefore, for many scientists and technicians, particularly those who are exposed to several different laboratory animal species and who perform a number of different tasks with the animals, measurements of potential exposure with filter capture and analysis techniques are probably of limited value. Indeed, this was judged to be the case both for the previous studies of ALA at ICI and this study.

It is thus still an assumption that the reduction in the incidence of ALA at ICI during the period from 1980 to $1984,{ }^{6}$ and the maintenance of this lower incidence in recent years, reported here, is due to the introduction of measures designed to reduce exposure to ani- mals. Although it has been suggested by others that the most heavily exposed people are the most likely to develop $\mathrm{ALA},{ }^{2}$ further prospective studies are needed to better define the relation between exposure levels (and patterns of exposure) and the incidence of the disease. Only then will it be possible to focus on the most appropriate management and engineering controls required to minimise the development of ALA. It is intriguing to note that in our study, the incidence of immunological sensitisation to laboratory animals is three to four times higher than the incidence of ALA. This may indicate that the improved working conditions, and the presumed lower exposures to animal allergens, may be sufficient to protect some people from becoming sensitised but that they may, for many people, simply avoid the development of symptoms and not sensitisation.

Our study has also looked into the controversial area of genetic susceptibility to ALA and specifically the effect of pre-existing atopy or sensitisation to laboratory animals. One fundamental problem is the lack of an agreed set of criteria to define atopy. Previous studies have used two broad definitions of atopy, personal history of allergy and skin prick test reactions to common environmental allergens. ${ }^{10}$ Although with either definition there seems to be an association between atopic diathesis and ALA, ${ }^{11}$ most of the studies reported have again been cross sectional and the significance of the finding is, therefore, debatable. In this study, and in our previous prospective study, ${ }^{6}$ a person was considered to be atopic if they had a positive skin test to grass pollens or house dust mite extract at the start of work with animals at ICI, a positive test being the development of a wheal of $\geqslant 4$ $\mathrm{mm}$ diameter. Although conservative, this definition resulted in an incidence of atopy of $46 \%$ among the 218 people initially recruited into this study, which is relatively high (the incidence of atopy in the general population is generally considered to be about $33 \%^{1}$ ).

We have again found a positive association between pre-existing atopy and the development of ALA. An atopic person is more likely to develop ALA than a non-atopic person, with an increased relative risk of between 4 and 8 at different times. An atopic person is also more likely to develop IgE antibodies to animal allergens, especially during the first year of employment when the increased relative risk is up to 7 . The table shows that the exclusion of atopic people from work with laboratory animals would have resulted in an incorrect decision in one third of the cases. Of the atopic people who would have been excluded from employment, $83 \%$ would not have developed ALA after one year and $74 \%$ would not have developed ALA after two years of exposure (fig 4). This confirms the conclusion that the exclusion of atopic people is difficult to justify. ${ }^{26}$

Perhaps not surprisingly, pre-existing sensitisation to laboratory animals also showed a strong positive association with the development of ALA, the relative risk being up to 8 . 
The exclusion of sensitised people would have resulted in only $13 \%$ incorrect decisions after two years (table).

Almost $50 \%$ of the sensitised people who would have been excluded would not have developed ALA after two years of exposure. Combination of sensitisation with atopy made little difference to the predictive value.

We think that these findings confirm the view that skin prick tests (and possibly also RAST analysis) usefully contribute to the medical assessment before employment. When atopic people and those already sensitised to laboratory animals are identified, they can be advised of their increased chance of developing ALA, particularly early in their work with animals, but they should not necessarily be excluded from this type of work. Consideration should be given to rejection of people who have a history of ALA, chronic skin disease, asthma, or other cardiorespiratory disorders, as these conditions do seem to make people much more susceptible to the induction or consequences of ALA than either atopy or the presence of IgE antibodies to laboratory animal allergens. ${ }^{1213}$

We acknowledge particularly the assistance of B Singleton who developed a computerised recall system to track people in the study. The dedication of the Occupational Health nurse at Alderley Park in completing the questionnaires and conducting the skin tests is recognised as is the careful laboratory work conducted by the staff of the Immunology Group at the Central Toxicology Laboratory.

1 Hunskaar S, Fosse RT. Allergy to laboratory mice and rats: a review of the pathophysiology, epidemiology and clinical aspects. Lab Anim 1990;24:358-74.

2 Kibby T, Powell G, Cromer J. Allergy to laboratory animals: a prospective and cross-sectional study. $\mathcal{f}$ Occup Med 1989;31:842-6.

3 Sjostedt L, Willers S. Predisposing factors in laboratory animal allergy: a study of atopy and environmental facanimal allergy: a study of atopy and
tors. $A m$ f Ind Med 1989;16:199-208.

4 Aoyama K, Ueda A, Manda F, Matsushita T, Ueda T, Yamauchi C. Allergy to laboratory animals: an epidemiological study. Br f Ind Med 1992;49:41-7.

5 Davies GE, Thompson AV, Niewola Z, et al. Allergy to laboratory animals: a retrospective and a prospective study. Br $\mathcal{\text { Ind }}$ Med 1983;40:442-9.

6 Botham PA, Davies GE, Teasdale EL Allergy to laboratory animals: a prospective study of its incidence and of tory anfuence of atopy on its development. $\mathrm{Br} \mathcal{F}$ Ind $\mathrm{Med}$ $1987 ; 44: 627-32$

7 Gordon S, Tee RD, Lowson D, Wallace J, Newman Taylor AJ. Reduction of airborne allergenic urinary proteins from

8 Eggleston PA, Newill CA, Ansari AA, et al. Task-related variation in airborne concentrations of laboratory animal allergens: studies with rat nl. $\mathcal{F}$ Allergy Clin Immunol 1989;84:347-52.

9 Price JA, Longbottom JL. Allergy to mice. I. Identification of two major mouse allergens (Agl and Ag3) and investigations of their possible origin. Clin Allergy 1987:17: 43-53.

10 Slovak AJM, Hill RN. Does atopy have any predictive value for laboratory animal allergy? A comparison of difvalue for laboratory animal allergy? A comparison of dif-
ferent concepts of atopy. $B r f$ Ind $M e d$ 1987;44:129-32.

11 Das R, Tager IB, Gamsky T, Scheneker MB, Royce S, Balmes JR. Atopy and airways reactivity in animal health Balmes JR. Atopy and airways reactivity in animal health
technicians. A pilot study. $f$ Occup Med 1992;34:53-60.

12 Lutsky I, Kalbfleisch JH, Fink JN. Occupational allergy to laboratory animals: employer practices. $\mathcal{f}$ Occup Med 1983;25:372-6.

13 Newill CA, Evans R, Khoury MJ. Pre-employment screening for allergy to laboratory animals - an epidemiological evaluation of its potential usefulness. 7 Occup Med 1986;28:1158-64. 